\title{
Development of Modified Selective Media to Differentiate Cryptococcus Species Complex and its Serotypes using Natural Materials
}

\author{
Gyu-Nam Park ${ }^{1}$, Hye-Ran Kim ${ }^{1}$, Dong-Jun $\mathrm{An}^{2}$, Hee-Sun Chae ${ }^{3}$ and Kyung-Soo Chang ${ }^{1, \uparrow}$ \\ ${ }^{I}$ Department of Clinical Laboratory Science, College of Health Sciences, \\ Catholic University of Pusan, Busan 46252, Korea \\ ${ }^{2}$ Animal and Plant Quarantine and Inspection Agency (QIA), Gimcheon-si, Gyeongsangbuk-do 39660, Korea \\ ${ }^{3}$ Seoul Metropolitan Government Research Institute of Public Health \& Environment, \\ Gwacheon, Gyeonggi 13818, Korea
}

\begin{abstract}
The formation of brown colonies due to phenol oxidase activity on classic agar media containing natural material extracts of Helianthus annuus or on medium containing L-3,4-dihydroxyphenylalanine has been used to identify Cryptococcus species complex. In this study, various natural materials were used to develop a modified medium and to identify five major serotypes of Cryptococcus species complex. Serotypes A, D, and A/D were pigmented on medium using Perilla frutescens var. japonica Hara (PerJ agar) after a three-day incubation. Serotypes B and C were pigmented on PerJ agar after four- and five-day incubations, respectively. Growth time and pigmentation of the five serotypes occurred more rapidly on PerJ agar than on the other media. In addition, colony morphology, size, and pigmentation were specific by serotype. In conclusion, PerJ agar should be used in clinic settings to identify Cryptococcus species complex and its serotypes rapidly.
\end{abstract}

Key Words: Cryptococcus species complex; HPLC; L-DOPA; Modified selective media; Serotype; Natural materials

\section{INTRODUCTION}

Encapsulated yeast of the genus Cryptococcus contains two major pathogenic species: Cryptococcus neoformans (C. neoformans) and Cryptococcus gattii (C. gattii). Among the more than 30 different species of Cryptococcus, only $C$. neoformans and $C$. gattii are considered major causative agents of cryptococcal infections. In particular, they are fatal, opportunistic pathogens that cause systemic fungal infection in human and animal hosts (Negroni, 2012). Cryptococcosis is one of the most common secondary infections in immune compromised hosts, particularly AIDS patients. Globally, there are approximately one million cases of meningitis each year in AIDS patients, resulting in approximately 624,700 deaths within three months after becoming infected with Cryptococcus species complex. As such, these fungi are defined as among the most important opportunistic fungal pathogens (Park et al., 2009).

Currently, Cryptococcus species complex are classified into two distinct species and three varieties, based on the different immunologic properties and epidemiology. C. neoformans (including two varieties: var. grubii and var. neoformans) is distributed worldwide, especially in bird droppings

* Received: February 14, 2017 / Revised: March 27, 2017 / Accepted: April 5, 2017

${ }^{\dagger}$ Corresponding author: Kyung-Soo Chang. Department of Clinical Laboratory Science, College of Health Sciences, Catholic University of Pusan, Busan 46252, Korea

Tel: +82-51-510-0565, Fax:+82-51-510-0568, e-mail: kschang@cup.ac.kr

(C) The Korean Society for Biomedical Laboratory Sciences. All rights reserved.

(C) This is an Open Access article distributed under the terms of the Creative Commons Attribution Non-Commercial License (http://creativecommons.org/licenses/by-nc/3.0/) which permits unrestricted non-commercial use, distribution, and reproduction in any medium, provided the original work is properly cited. 
and nests, while $C$. gattii has a more geographically restricted distribution and is prevalent in tropical and subtropical regions (Negroni, 2012). Although isolation and identification of these fungal pathogens from clinical sources is very important for patients, as cryptococcosis is a lifethreatening infection, they are time-consuming and complicated processes. Diagnosis of Cryptococcus species complex from clinical materials is generally based on the observation capsule of yeasts by direct microscopy; culture and biochemical tests are subsequently required for isolation and identification of these fungal pathogens (Negroni, 2012). Classic media containing natural material extracts (such as Guizotia abyssinica [niger seed agar] or Helianthus annuus [sunflower seed agar]) or chemicals (such as caffeic acid or L-3,4-dihydroxyphenylalanine [L-DOPA]) are commonly used as the primary selective culture media for detecting typical brown pigment formation due to phenyl oxidase activity. These selective culture media are useful tools for isolating Cryptococcus species complex from environmental and clinical materials (Eisenman et al., 2011; Menezes et al., 2011). However, these selective culture media have some limits. For instance, the incubation step of Cryptococcus species complex takes too long to detect brown pigment formation, and it is very difficult to distinguish among the species of Cryptococcus species complex.

Therefore, in this study, we attempted to develop modified selective culture media by using various affordable natural material extracts. In addition, we compared the growth of Cryptococcus species complex strains on ten different natural material-based media.

\section{MATERIALS AND METHODS}

\section{Yeast strains}

In this study, four reference strains were purchased from the American Type Culture Collection (ATCC). ATCC 66031 (serotype D) and 48184 (serotype A/D) strains were used as reference strains for $C$. neoformans var. neoformans and ATCC MYA-4560 (serotype B) and 32608 (serotype C) strains were used as reference strains for $C$. gattii. One clinical strain, which was identified as $C$. neoformans var. grubii (serotype A) in a previous study, was used as a reference strain for $C$. neoformans var. grubii (Chae et al., 2012). Non-Cryptococcus species complex strains - three Candida species (C. albicans, C. tropicalis, and C. glabrata) isolates -were provided by General Hospitals in Busan. And we used seventeen environmental samples of pigeon droppings which are confirmed as Cryptococcus species complex positive samples in previous study (Chae et al., 2012). All isolates were identified using the Vitek2 yeast identification system (bioMerieux, Marcy l'Etoile, France) and the genotyping method used in previous studies (Chae et al., 2012). All yeast strains were stored at $-70^{\circ} \mathrm{C}$ in a deep freezer until used.

\section{Primary culture of yeast strains}

To prepare the yeast culture suspensions, all yeast strains were recovered from frozen glycerol stocks. Briefly, a yeast suspension of $100 \mu \mathrm{l}$ was inoculated onto Sabouraud dextrose agar (SDA). All plates were incubated in the dark at $25^{\circ} \mathrm{C}$ under humid conditions for five days. The cultures were observed daily to monitor growth and formation of pure colonies. After five days, the recovered pure colonies of each strain were transferred into Sabouraud dextrose broth and incubated on a shaker for $24 \sim 48$ hours at $25^{\circ} \mathrm{C}$.

\section{Preparation of SSA and various natural material media}

Sunflower (Helianthus annuus seed agar (SSA), which is used as a typical selective agar for Cryptococcus species complex, was prepared as described previously [Khan et al., 2004]. Briefly, the natural materials were ground as finely as possible with a grinder. Then, $25 \mathrm{~g}$ of the ground natural materials were boiled in $500 \mathrm{ml}$ of distilled water (DW) for $30 \mathrm{~min}$ and filtered through several layers of gauze. Next, $7.5 \mathrm{~g}$ of Bacto agar, $0.5 \mathrm{~g}$ of glucose, $0.5 \mathrm{~g}$ of $\mathrm{KH}_{2} \mathrm{PO}_{4}$, and $0.5 \mathrm{~g}$ of creatinine were added, and the volume was readjusted to $500 \mathrm{ml}$. The mixture was autoclaved at $121^{\circ} \mathrm{C}$ for $15 \mathrm{~min}$, after which the medium was dispensed into $50 \mathrm{~mm}$ diameter petri dishes (10 $\mathrm{ml}$ per plate). To prepare the modified natural material media, various dried natural materials (including Sesamum indicum, Heukimja, Perilla frutescens var. japonica Hara, Glycine max [L.]) Merrill, Pinus koraiensis, Ricinus communis L., Arachis hypogaea, Juglans nigra L., and Juglans sinensis Dode) were purchased from 
a local market. The same method used to prepare the SSA was followed.

\section{Culture of yeast strains in various natural material media}

All of the yeast suspensions were cultured on various natural material media. Five strains of Cryptococcus species complex (ATCC 66031, 48184, MYA-4560, 32608, and one clinical isolate) and four isolates of non-Cryptococcus species (C. albicans, C. tropicalis, and C. glabrata) were inoculated on SSA and various other natural material media. The plates were incubated in the dark at $25^{\circ} \mathrm{C}$ under humid conditions and observed at 12-hour intervals. All experiments were performed in triplicate.

\section{Culture of environmental samples in various natural material media}

Culture method was described in previous study (Chae et al., 2012). Briefly, each samples were suspended in phosphate buffered saline (PBS) by vortexing, and centrifuged. Then $100 \mu \mathrm{l}$ of aliquot from supernatant were inoculated onto SSA and various other natural material media. The plates were incubated in the dark at $25^{\circ} \mathrm{C}$ under humid conditions for $2 \sim 8$ days. All plates were examined daily to observe the specific color of yeast-form colonies.

\section{HPLC analysis for natural material extract}

To perform high-performance liquid chromatography (HPLC) analysis, aqueous natural material extract samples were prepared. The natural material extract samples were extracted from ground natural materials with different two solvents - ethanol and DW. The extraction process using DW was performed by boiling ten dried natural materials $(5.0 \mathrm{~g})$ in DW $(100 \mathrm{ml})$ for one hour. The extraction and sample preparation processes using ethanol were as follows. Two dried natural materials (Helianthus annuus and Perilla frutescens var. japonica Hara) were dissolved in $80 \%$ ethanol (100 ml) and stored for 24 hours with the help of a shaker set at $25^{\circ} \mathrm{C}$. The natural material extracts were filtered through several layers of gauze, after which the extract was centrifuged at 2,500 $\times g$ and the supernatant was decanted.

The standard material (L-DOPA) was purchased from Sigma-Aldrich (USA). All natural material extract samples and standard material were obtained at a professional facility (Koptri, Seoul, Korea). All samples were filtered through a $0.45 \mu \mathrm{m}$ PTFE filter and analyzed with a Warters 600 pump, Warters 717 automatic sample injection system, Waters PDA detector, and Empower software. A TSKgel ODS-100V column $(4.6 \times 250 \mathrm{~mm})$ was used at a flow rate of $1.0 \mathrm{ml} /$ $\min$. The temperature of the column was set at $35^{\circ} \mathrm{C}$, and the injection volume was $10 \mu \mathrm{l}$.

\section{RESULTS}

\section{Phenotype characteristics of yeast strains on various natural material-based media}

Five strains of Cryptococcus species complex (ATCC 66031, 32608, MYA-4560, 48184, and one clinical strain) and three clinical isolates of Candida species (C. albicans, C. glabrata, and C. tropicalis) were cultured on SDA and ten different natural material-based media, respectively. Three clinical isolates of Candida species produced no pigment (Table 2). Only white colonies were observed on all of the natural material-based media, with the exception of Pk (using Pinus koraiensis) and JjD (using Juglans nigra L. and Juglans sinensis Dode) agar (no growth). Table 1 shows pigment formation by five strains of Cryptococcus species complex on different natural material-based media. On the typical SSA selective media, all strains of Cryptococcus species complex were differentiated from the white colonies of Candida species by specific brown pigment formation. However, no differences were detected between serotypes of Cryptococcus species complex.

On the SeI (using Sesamum indicum) agar, five strains of Cryptococcus species complex were classified into two types of colonies according to colony color (light brown [LB] and white [W]). Serotypes A, B, D, and A/D produced LB pigment, whereas serotype $\mathrm{C}$ was W. On GIM (using Glycine max [L.] Merrill) agar, the strains were classified into three types of colonies according to colony color (dark yellow [DY], beige [Be], and W). Serotypes A, D, and A/D produced DY pigment, whereas serotype $\mathrm{B}$ was Be. The colony color of serotype C remained the same (W). On RiC (using Ricinus communis L.) agar, the strains were classified into two types of colonies according to colony color (DY and 
Table 1. Different pigment formations on modified natural material-based culture agar according to serotypes of Cryptococcus species complex

\begin{tabular}{|c|c|c|c|c|c|}
\hline \multirow[b]{2}{*}{ Natural materials } & \multicolumn{5}{|c|}{ Cryptococcus species complex } \\
\hline & Serotype A & Serotype B & Serotype C & Serotype D & Serotype A/D \\
\hline Helianthus annuus $L$. & B & B & B & B & B \\
\hline Sesamum indicum & LB & LB & $\mathrm{W}$ & LB & LB \\
\hline Heukimja & LB & LB & $\mathrm{W}$ & LB & LB \\
\hline $\begin{array}{l}\text { Perilla frutescens var. } \\
\text { japonica Hara }\end{array}$ & B & B & $\mathrm{B}$ & B & B \\
\hline Glycine max (L.) Merrill & DY & $\mathrm{Be}$ & $\mathrm{W}$ & DY & DY \\
\hline Ricinus communis $L$. & DY & DY & $\mathrm{W}$ & DY & DY \\
\hline Arachis hypogaea & LY & LY & $\mathrm{W}$ & LY & LY \\
\hline Prunus dulcis & LY & LB & W & LB & LY \\
\hline Pinus koraiensis & LY & No growth & $\mathrm{W}$ & No growth & No growth \\
\hline $\begin{array}{l}\text { Juglans nigra L., Juglans } \\
\text { sinensis Dode }\end{array}$ & No growth & No growth & No growth & No growth & No growth \\
\hline
\end{tabular}

*light brown; LB, brown; B, white; W, dark yellow; DY, light yellow; LY, and beige; Be.

Table 2. Pigment formations of Candida species on modified natural material-based culture agar

\begin{tabular}{lccc}
\hline \hline \multirow{2}{*}{ Natural materials } & \multicolumn{3}{c}{ Candida species } \\
\cline { 2 - 4 } & C. albicans & C. tropicalis & C. glabrata \\
\hline Helianthus annuus L. & $\mathrm{W}$ & $\mathrm{W}$ & $\mathrm{W}$ \\
Sesamum indicum & $\mathrm{W}$ & $\mathrm{W}$ & $\mathrm{W}$ \\
Heukimja & $\mathrm{W}$ & $\mathrm{W}$ & $\mathrm{W}$ \\
Perilla frutescens var. japonica Hara & $\mathrm{W}$ & $\mathrm{W}$ & $\mathrm{W}$ \\
Glycine max (L.) Merrill & $\mathrm{W}$ & $\mathrm{W}$ & $\mathrm{W}$ \\
Ricinus communis L. & $\mathrm{W}$ & $\mathrm{W}$ & $\mathrm{W}$ \\
Arachis hypogaea & $\mathrm{W}$ & $\mathrm{W}$ & $\mathrm{W}$ \\
Prunus dulcis & $\mathrm{W}$ & $\mathrm{W}$ & $\mathrm{W}$ \\
Pinus koraiensis & No growth & No growth & No growth \\
Juglans nigra L., Juglans sinensis Dode & No growth & No growth & No growth \\
\hline
\end{tabular}

*light brown; LB, brown; B, white; W, dark yellow; DY, light yellow; LY, and beige; Be.

W). Serotypes A, B, D, and A/D produced DY pigment, whereas serotype $\mathrm{C}$ was W. On ArH (using Arachis hypogaea) agar, the strains were classified into two types of colonies according to colony color (light yellow [LY] and W). Serotypes A, B, D, and A/D produced LY pigment, whereas serotype $\mathrm{C}$ was white. On $\operatorname{PrD}$ (using Prunus dulcis) agar, the strains were classified into three types of colonies according to colony color (LY, LB, and W) (Fig. 1A). Serotypes $\mathrm{A}$ and $\mathrm{A} / \mathrm{D}$ produced $\mathrm{LY}$ pigment, whereas serotypes
$\mathrm{B}$ and $\mathrm{D}$ were LB. The colony color of serotype $\mathrm{C}$ remained the same (W). On SeI, GlM, RiC, ArH, and PrD agar, slight changes in colony color were observed in almost all Cryptococcus spp. cultured media. However, brown or other specific pigment formation were not observed in all strains of Cryptococcus species complex.

On Heu (using Heukimja) agar, the strains were classified into two types of colonies according to colony color (LB and W) (Fig. 1B). Serotypes A, B, D, and A/D produced 
A
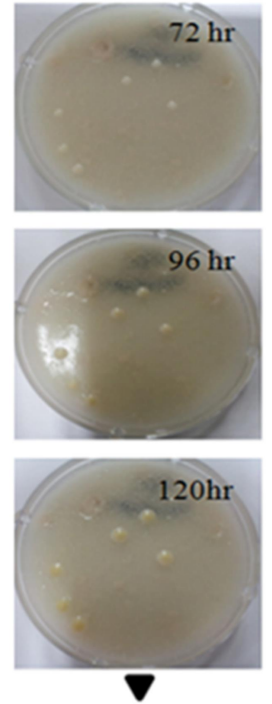

Serotype A
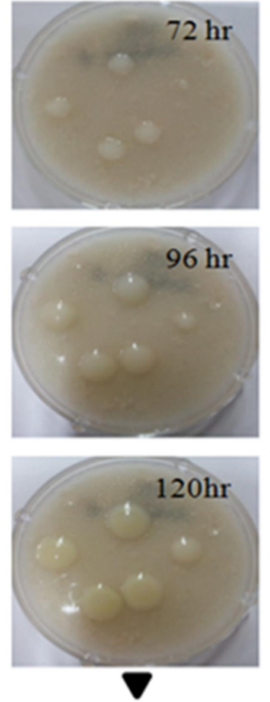

Serotype B
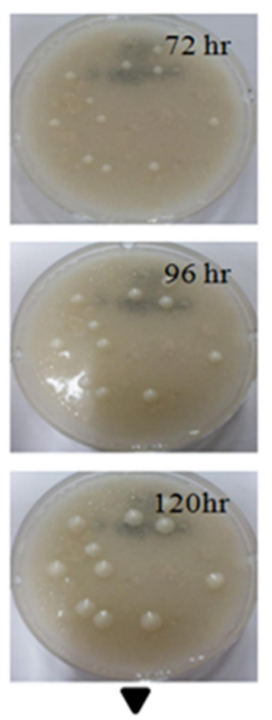

Serotype C
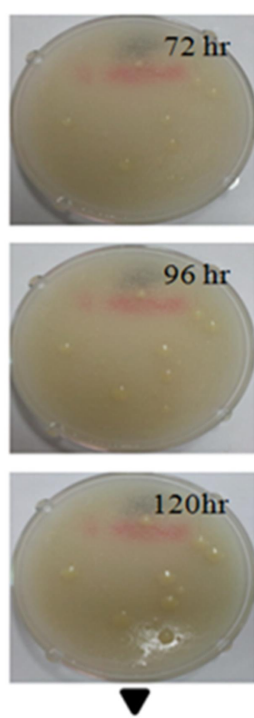

Serotype D
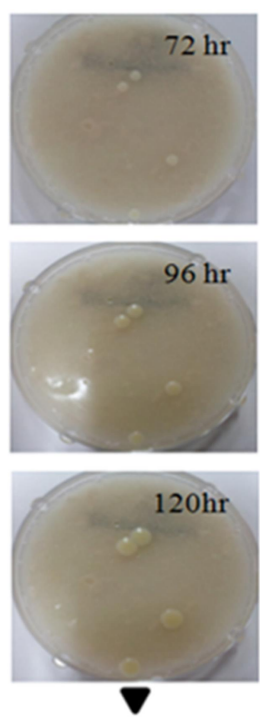

Serotype AVD

B

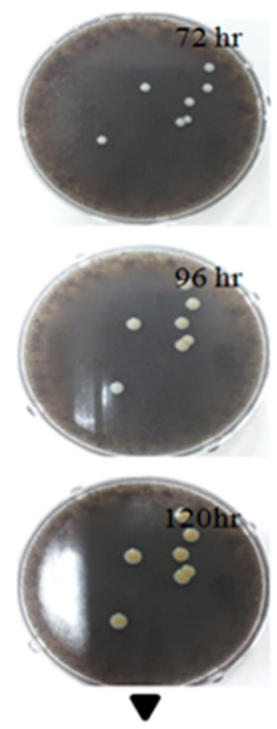

Serotype A
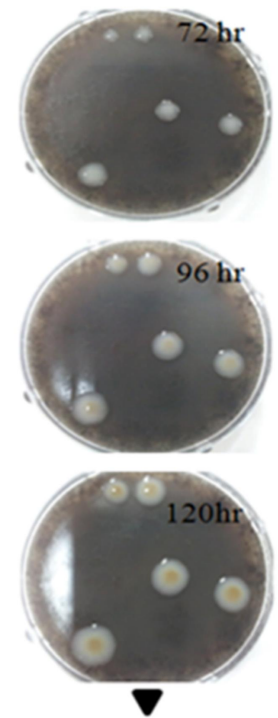

Serotype B
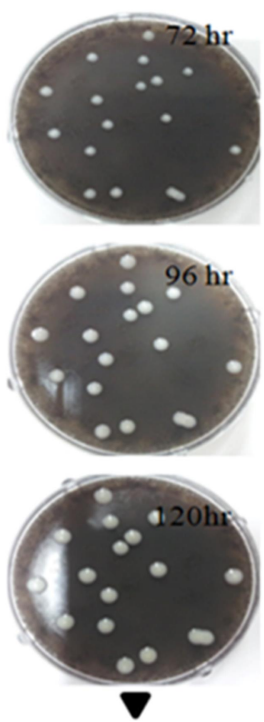

Serotype C
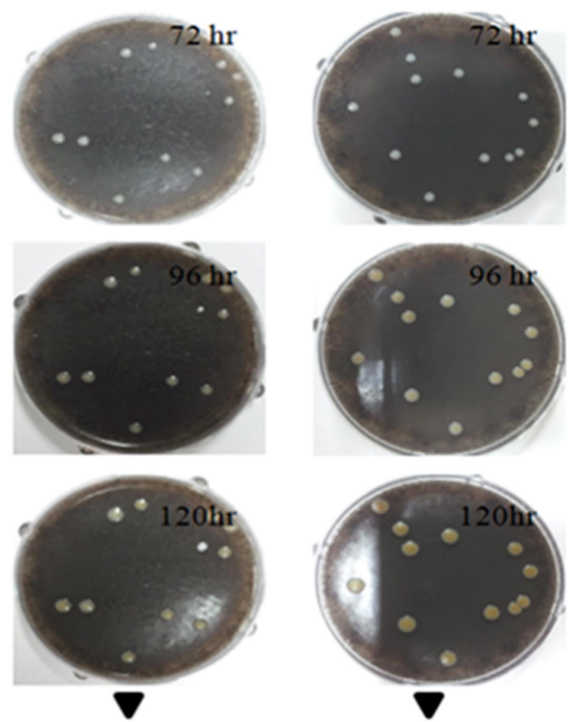

Serotype D

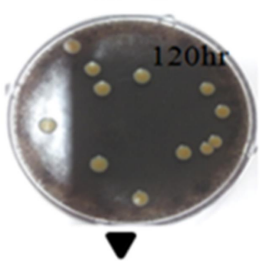

Serotype A/D

Fig. 1. Pigment formation of Cryptococcus species complex on natural material-based media according to incubation times. (A) Pigment formation of Cryptococcus species complex on PrD agar according to incubation times. (B) Pigment formation of Cryptococcus species complex on Heu agar according to incubation times.

LB pigment, whereas serotype C was white. Unusually, serotype B on Heu media was observed to have a different pigment formation than the other serotypes. LB pigment was observed exclusively in the center zone of the colony. This formation, which appeared in all serotype B colonies cultured on Heu media, did not change over incubation time.

On PerJ (using Perilla frutescens var. japonica Hara) agar, brown pigment was observed in all strains of Cryptococcus spp. cultured media (Fig. 2). Colony growth and pigment formation occurred more rapidly than on the typical 


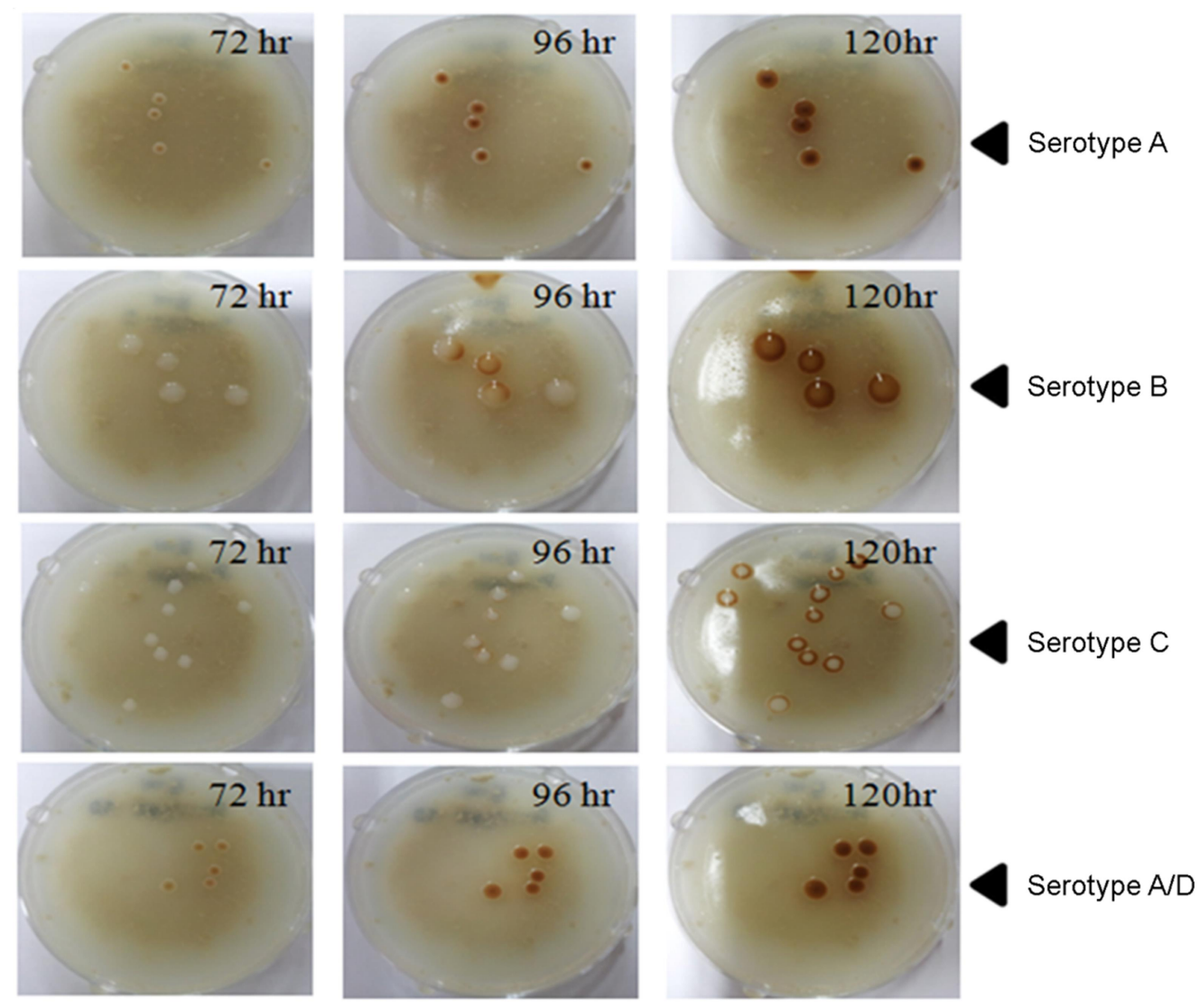

Fig. 2. Specific pigmentation of Cryptococcus species complex on PerJ agar according to incubation times.

selective medium, SSA. After incubating for 48 hours, only white colonies were observed on SSA, but brown pigmented colonies were observed in serotype A, D, and A/D strains cultured on PerJ agar. Furthermore, a specific pigment formation was observed in serotype $\mathrm{B}$ and $\mathrm{C}$ cultured mediabrown pigment was produced in the rim of the colony first - which was different from the other serotypes on PerJ agar. On Pk and JjD agar, colony growth was not observed in almost all yeast strains on cultured media. In particular, colony growth was not observed at all on JjD media.

\section{Isolation of Cryptococcus species complex strains from environmental samples on various natural material- based media}

Specific brown pigment yeast-form colonies were observed on the SSA and PerJ agar. To identification of brown yeast-form isolates, we performed genotyping by using ITS sequencing (Chae et al., 2012). As a result, all brown yeastform isolates were identified as Cryptococcus neoformans. And we subcultured each brown isolates on PerJ agar for detection of the pigment formation pattern. The pigmentation pattern of all isolates was same as serotypes $\mathrm{A}, \mathrm{D}$, and $\mathrm{A} / \mathrm{D}$ strains.

\section{HPLC analysis to quantify L-DOPA}

L-DOPA is one of the typical exogenous substrates required to synthesize melanin pigment in Cryptococcus species complex. To quantify L-DOPA content in ten different natural material extract samples, HPLC analysis was performed at a professional facility. To evaluate the calibration curve for L-DOPA, a standard solution of L-DOPA was prepared based on a concentration range of $1 \sim 100 \mathrm{mg} /$ $\mathrm{L}(1,10,50$, and $100 \mathrm{mg} / \mathrm{L}[\mathrm{ppm}])$ and evaluated at $305 \mathrm{~nm}$. As a result, a sharp peak was detected at a retention time of 
Table 3. The HPLC analysis of dried natural material extracts

\begin{tabular}{lcccc}
\hline \hline \multicolumn{1}{c}{ Extracts } & $\begin{array}{c}\text { Area } \\
\left(\mu \mathrm{V}^{*} \mathrm{sec}\right)\end{array}$ & \% Area & $\begin{array}{c}\text { Height } \\
(\mu \mathrm{V})\end{array}$ & $\begin{array}{c}\text { Retention time } \\
(\mathrm{min})\end{array}$ \\
\hline Standard, L-3,4-dihydroxyphenylalanine & 353044 & 100.0 & 21505 & 2.28 \\
Helianthus annuus L. (DW) & - & - & - & - \\
Helianthus annuus L. (ethanol) & - & - & - & - \\
Perilla frutescens var. japonica Hara (DW) & 402556 & 100.0 & 14223 & 2.3 \\
Perilla frutescens var. japonica Hara (ethanol) & 104159 & 100.0 & 3506 & 2.89 \\
Sesamum indicum & 270347 & 100.0 & 10114 & 2.21 \\
Heukimja & 134417 & 100.0 & 5327 & 2.24 \\
Glycine max (L.) Merrill & 764313 & 100.0 & 25208 & 2.31 \\
Ricinus communis L. & 885919 & 100.0 & 33015 & 2.21 \\
Arachis hypogaea & 575419 & 100.0 & 18995 & 2.21 \\
Prunus dulcis & - & - & - & - \\
Pinus koraiensis & 799158 & 100.0 & 31088 & 2.18 \\
Juglans nigra L., Juglans sinensis Dode & 664361 & 100.0 & 19404 & 2.29 \\
\hline
\end{tabular}

$2.28 \mathrm{~min}$. The coefficient of correlation $\left(\mathrm{R}^{2}\right)$ was 0.999 , and the equation of the calibration curve was $\mathrm{Y}=3576 \mathrm{X}-2282.4$. Consequently, good linearity of the calibration curve for L-DOPA was established (data not shown). The quantity of L-DOPA content in the natural material extract samples was subsequently analyzed, and no L-DOPA was detected in any of the samples. In other words, the retention time of the peaks for the natural material extract samples was different from that of the standard compound, L-DOPA (Table 3). This result suggests that the ten different natural materialbased media did not contain L-DOPA.

\section{DISCUSSION}

Culture media for microorganisms have played important roles in the primary isolation and identification of microorganisms from various sources in many clinics and laboratory fields. This method is simple, cost effective, and a useful tool for diagnosing infectious diseases. Many typical selective and differential media have specific indicators to differentiate among microorganism mixtures, such as certain inhibitory agents (e.g., antibiotics, chemicals, $\mathrm{pH}$ ) and substrates. In particular, pigment formation is one of the typical indicators on culture media for isolating and identifying microorganisms. For instance, yeast strains obtained from environmental sources have been classified easily using colony color type (brown type, white type, beige type, and pink type) on SSA (Chae et al., 2012). These results suggest that good selective and differential media can be considered to be useful screening tools for identifying microorganisms.

The encapsulated pathogenic yeast Cryptococcus species complex has the ability to produce a specific dark brown pigment called melanin on media containing certain diphenolic compounds, such as L-DOPA. Cryptococcus species complex cannot produce melanin pigment without certain exogenous substrates (Casadevall et al., 2003; Eisenman et al., 2011; Garcia-Rivera et al., 2005). Based on this idea, several modified culture media were developed for the rapid identification of Cryptococcus species complex, such as mustard seed, chili pepper, Pinus halepensis seed, Eggplant, benomyl bird seed and blackberry agar (Menezes et al., 2011; Mseddi et al., 2011; Nandhakumar et al., 2006; Pedroso et al., 2007; Pham et al., 2014; Sengul et al., 2014). However, there are some limits. Almost all previously developed selective media for Cryptococcus species complex have focused on rapid pigmentation, or melanization. Of course, it is an important request, but is not everything. Currently, Cryptococcus species complex are classified into two species and five major serotypes. It is important to identify Cryptococcus species complex accurately on selective media. As 
such, we attempted to develop modified selective culture media to differentiate among Cryptococcus species using various affordable natural material extracts.

The results indicated that SeI, GlM, RiC, ArH, PrD, Pk, and $\mathrm{JjD}$ agar were not suitable for use as selective media for Cryptococcus species complex. On those media, either no specific pigmentation was observed (SeI, GIM, RiC, ArH, and PrD agar) or not all of the cryptococcal strains grew well ( $\mathrm{Pk}$ and $\mathrm{JjD}$ agar). On the Heu agar, a specific pigmentation pattern was observed in only the serotype $B$ strain (Fig. 1A), and this medium was also not suitable. On the other hand, PerJ agar was a specific medium for Cryptococcus species complex. On PerJ agar, a brown pigment formation on the colony was observed more rapidly than on the typical medium, SSA (Fig. 2). In addition, the various Cryptococcus species had different brown pigmentation patterns. In serotypes A, D, and A/D of the strains cultured on PerJ agar, brown pigment was produced in the center of the colony first, but it was produced in the rim of colony first in serotypes B and C. These results suggest that PerJ agar is a useful medium for the rapid identification and differentiation of the serotypes of Cryptococcus species complex.

It is well known that certain exogenous substrates are required to produce the brown pigment of Cryptococcus species complex on media. Therefore, HPLC analysis was performed to evaluate L-DOPA content in the natural material extracts. The retention times of all of the natural material extracts were different from that of the standard compound, L-DOPA (Table 3), indicating that none of the samples extracted from the ten different natural materials contained L-DOPA. These results suggest that the brown pigmentation mechanism of Cryptococcus species complex on SSA or PerJ agar might be different on typical L-DOPAcontaining media. Thus, further studies regarding the exact brown pigmentation mechanism of Cryptococcus species complex on PerJ agar are needed. One problem with making PerJ agar is that it is a complicated procedure. Khan and colleagues previously described a simplified SSA (without creatinine and $\mathrm{KH}_{2} \mathrm{PO}_{4}$ ) (Khan et al., 2004); therefore, we attempted to make simplified PerJ agar. Five strains of Cryptococcus species complex were cultured on the simpli- fied PerJ agar under the same conditions, and brown pigment was produced well (data not shown).

In this study, we attempted to develop modified selective media for Cryptococcus species complex in order to more rapidly identify and distinguish among their serotypes. Modified PerJ agar is a useful medium that is made with affordable ingredients. It is a simple and cost-effective medium for differentiating Cryptococcus species complex from clinical and environmental sources.

\section{ACKNOWLEDGEMENTS}

This study was supported by a grant (Z-AD20-201112-01) from the Animal, Plant \& Fisheries Quarantine and Inspection Agency (QIA), Ministry of Food, Agriculture, Forestry and Fisheries, Republic of Korea, in 2011-2012. This study was supported by BB21 (Brain Busan21), Republic of Korea.

\section{CONFLICT OF INTEREST}

There was no conflict of interests between all authors.

\section{REFERENCES}

Casadevall A, Rosas AL, Nosanchuk JD. Melanin and virulence in Cryptococcus neoformans. Current Opinion in Microbiology. 2003. 3: 354-358.

Chae HS, Park GN, Kim SH, Jo HJ, Kim JT, Jeoung HY, An DJ, Kim NH, Shin BW, Kang YI, Chang KS. Rapid direct identification of Cryptococcus neoformans from pigeon droppings by nested PCR using CNLAC1 gene. Poultry Science. 2012. 91: 1983-1989.

Chae HS, Jang GE, Kim NH, Son HR, Lee JH, Kim SH, Park GN, Jo HJ, Kim JT, Chang KS. Classification of Cryptococcus neoformans and yeast-like fungus isolates from pigeon droppings by colony phenotyping and ITS genotyping and their seasonal variations in Korea. Avian Diseases. 2012. 56: 58-64.

Eisenman HC, Chow SK, Tsé KK, McClelland EE, Casadeval A The effect of L-DOPA on Cryptococcus neoformans growth and gene expression. Virulence. 2011. 2: 329-336.

Garcia-Rivera J, Eisenman HC, Nosanchuk JD, Aisen P, Zaragoza O, Moadel T, Dadachova E, Casadevall A. Comparative analysis of Cryptococcus neoformans acid-resistant particles generated from pigmented cells grown in different laccase 
substrates. Fungal Genetics and Biology. 2005. 42: 989-998.

Khan ZU, Ahmad S, Mokaddas E, Chandy R. Simplified sunflower (Helianthus annuus) seed agar for differentiation of Candida dubliniensis from Candida albicans. Clinical Microbiology and Infection. 2004. 10: 590-592.

Menezes Rde P, Penatt MP, Pedroso Rdos S. Different culture media containing methyldopa for melanin production by Cryptococcus species. Revista da Sociedade Brasileira de Medicina Tropical. 2011. 44: 591-594.

Mseddi F, Sellami A, Sellami H, Cheikhrouhou F, Makni F, Ayadi A. Two new media Pinus halepensis seed agar and blackberry agar for rapid identification of Cryptococcus neoformans. Mycoses. 2011. 54: 350-353.

Nandhakumar B, Kumar CP, Prabu D, Menon T. Mustard seed agar, a new medium for differentiation of Cryptococcus neoformans. Journal of Clinical Microbiology. 2006. 44: 674.

Negroni R. Cryptococcosis. Clinics in Dermatology. 2012. 30: 599-609.

Park BJ, Wannemuehler KA, Marston BJ, Govender N, Pappas PG, Chiller TM. Estimation of the current global burden of cryptococcal meningitis among persons living with HIV/ AIDS. AIDS. 2009. 23: 525-530.

Pedroso RS, da Costa KR, Ferreira JC, Candido RC. Evaluation of melanin production by Cryptococcus species in four different culture media. Revista da Sociedade Brasileira de Medicina Tropical. 2007. 40: 566-568.
Pham CD, Ahn S, Turner LA, Wohrle R, Lockhart SR. Development and validation of benomyl birdseed agar for the isolation of Cryptococcus neoformans and Cryptococcus gattii from environmental samples. Medical Mycology. 2014. 52: 417-421.

Shin KS, Jo MY, Hon SB. Evaluation of the antibacterial effects of Phellinus baumii extract on methicillin-resistant staphylococcus aureus by using broth microdilution based on a colorimetric method. Biomedical Science Letters. 2016. 22: 147 -173 .

Sengul M, Ergin C, Kartal T. Evaluation of a new medium, eggplant (Solanum melongena) agar as a screening medium for Cryptococcus neoformans in environmental samples. Mikrobiyoloji Bulteni. 2014. 48: 292-299.

Yoo YB, Kim SS, Kim YK, Kim SH. Evaluation and optimization of a serum-based minimum inhibitory concentration assay to caspofungin in Candida albicans clinical isolates. Biomedical Science Letters. 2016. 22: 174-183.

http://dx.doi.org/10.15616/BSL.2017.23.2.64

Cite this article as: Park GN, Kim HR, An DJ, Chae HS, Chang KS. Development of Modified Selective Media to Differentiate Cryptococcus Species Complex and its Serotypes using Natural Materials. Biomedical Science Letters. 2017. 23: 64-72. 